\title{
Potential of artificial intelligence to accelerate diagnosis and drug discovery for COVID-19
}

\author{
Indira Mikkili ${ }^{\text {Corresp., } 1}$, Abraham Peele ${ }^{1}$, Chinna Venkateswrulu Thirupati ${ }^{1}$, Drkodali Vidya Prabhakar Kodali ${ }^{2}$, \\ Deepika Sri Singh Macamdas ${ }^{1}$, Krupanidhi Sreerama ${ }^{1}$ \\ ${ }^{1}$ Biotechnology, Vignan's Foundation for Science Technology \& Research, Guntur, Andhra Pradesh, India \\ 2 Biotechnology, Vikrama Simhapuri University, Nellore, Andhra Pradesh, India \\ Corresponding Author: Indira Mikkili \\ Email address: indu221007@gmail.com
}

The corona virus disease (COVID-19) pandemic has caused havoc worldwide. The tests currently used to diagnose COVID-19 are based on real time reverse transcription polymerase chain reaction (RT-PCR), computed tomography medical imaging techniques and immunoassays. It takes 2 days to obtain results from the RT-PCR test and also shortage of test kits creating a requirement for alternate and rapid methods to accurately diagnose COVID-19. Application of artificial intelligence technologies such as internet of things, machine learning tools and big data analysis to COVID-19 diagnosis could yield rapid and accurate results. The neural networks and machine learning tools can also be used to develop potential drug molecules. Pharmaceutical companies face challenges linked to the costs of drug molecules, research and development efforts, reduced efficiency of drugs, safety concerns and the conduct of clinical trials. In this review, relevant features of artificial intelligence and their potential applications in COVID-19 diagnosis and drug development are highlighted. 
1 Potential of artificial intelligence to accelerate diagnosis and drug discovery for COVID-19

2 M. Indira $^{1^{*}}$, Abraham Peele $^{1}$, T. C. Venkateswarulu ${ }^{1}$, K. Vidya Prabhakar ${ }^{2}$, M. Deepika Sri Singh ${ }^{1}$, 3 S. Krupanidhi ${ }^{1}$

4

$51^{1} 1^{*}$ Department of Biotechnology, Vignan's Foundation for Science, Technology \& Research, 6 Vadlamudi-522213, Andhra Pradesh, India.

7 2, Department of Biotechnology, Vikrama Simhapuri University, Nellore-524004, Andhra 8 Pradesh, India.

Corresponding Author: Dr. M. Indira, Associate Professor, Department of Biotechnology, Vignan's Foundation for Science, Technology \& Research, Vadlamudi-522213, Andhra Pradesh, India. Email: indu221007@gmail.com

\section{ABSTRACT}

The corona virus disease (COVID-19) pandemic has caused havoc worldwide. The tests currently used to diagnose COVID-19 are based on real time reverse transcription polymerase chain reaction (RT-PCR), computed tomography medical imaging techniques and immunoassays. It takes 2 days to obtain results from the RT-PCR test and also shortage of test kits creating a requirement for alternate and rapid methods to accurately diagnose COVID-19. Application of artificial intelligence technologies such as internet of things, machine learning tools and big data analysis to COVID-19 diagnosis could yield rapid and accurate results. The neural networks and machine learning tools can also be used to develop potential drug molecules. Pharmaceutical companies face challenges linked to the costs of drug molecules, research and development efforts, reduced efficiency of drugs, safety concerns and the conduct of clinical trials. In this review, relevant features of artificial intelligence and their potential applications in COVID-19 diagnosis and drug development are highlighted. 


\section{INTRODUCTION}

The COVID-19 pandemic is a worldwide health crisis. The causative agent for COVID-19 disease is severe acute respiratory syndrome coronavirus 2 (SARS-CoV-2) (Singhal, 2020). Various vaccines and drugs are being tested in trials for the treatment of COVID-19 disease and released in to the market for emergency purpose. SARS-CoV-2 infects the respiratory tract, mainly the lungs that leads to acute respiratory syndrome and finally death in severely ill and co-morbid patients. One challenge facing the health care workers is identifying people with COVID-19 (Wu et al., 2020). The disease is diagnosed using the test reverse transcriptase polymerase chain reaction (RT-PCR), which is the preferred method. However, the time taken to run the test and return the results is 2 days. Furthermore, there is an under-supply of RT-PCR test kits, lack of advanced technologies limits the disease identification and drug development process (Tahamtan \& Ardebili, 2020). An advanced and accurate diagnosis tool that yields rapid results is required (Carter et al., 2020). Artificial intelligence (AI) comprises advanced cognitive systems that are progressing rapidly across the world (Ahuja, 2019). AI algorithms can be used to enhance the findings from chest computed tomography (CT) imaging (Hosny et al., 2018) that enable the rapid diagnosis of a disease (Mei et al., 2020). Considering the public health perspective and the need to quickly isolate patients with COVID-19, chest CT is used for diagnostic imaging due to its sensitivity than chest radiography (Xu et al., 2020). There are few articles related to the detailed methods for detection of SARS CoV-2 and drug discovery using AI. AI tools and software can be used for the development of drugs in the pharmaceutical industry (Agarwal, 2018). Machine learning is a fundamental AI tool that uses algorithms to recognize various patterns in input data collected for disease as well as drug identification (Jiang et al., 2017). Within the machine learning, the subfield of deep learning involves neural networks that play an important role in collecting the input data and generating the output data (Nguyen et al., 2019). Artificial neural networks include recurrent neural networks, multilayer perception and convolutional neural networks (Nguyen et al., 2019). A convolutional network consists of a dynamic system with local connections characterized by its biological system modeling, use of images, topology, processing of complex brain functions, recognitions, signal processing and video processing (Abiodun et al., 2018). Multilayer perception consists of pattern recognition, identification of process and controls in a single direction only (Abiodun et al., 2019). A recurrent neural network consists of a closed loop that memorizes and stores information (Abiodun et al., 2019). In traditional drug discovery 
61 method, the first step is the target (disease) validation, followed by in vitro assays, screening of 62 compounds, lead identification, preclinical trails, clinical trials (Phase I, II, III and IV) and finally approval of drugs for clinical use (Riaz et al., 2020). The entire process takes 10-15 years to develop a potential drug molecule for disease therapy (Mohs et al., 2017). With AI, drug discovery starts with prediction of a target protein's role in disease, design of in silico compounds from libraries, novel target identification, prediction of structure-activity relationship, prediction of ADMET properties, drug repurposing, selection of patient population for clinical trials to increase success rates, pharmacovigilance, observation of adverse effects and interrogation of transcriptomic data (Paul et al., 2021). In every step in the development of potential lead molecules for disease therapy, AI plays an important role in minimizing the time required to produce good results (Bohr \& Memarzadeh, 2020). Machine learning tools and software helps in identifying specific target virtual molecules and optimizing the efficacy and safety of the drug molecules in human population. AI reduces the time for identification of potential target molecules virtually compared with the synthesis of multiple compounds used for in vitro and in vivo assays (Paul et al., 2021). Currently the health sector is facing the problems related to drugs and therapies for emerging diseases where nine out of ten drugs will fail in clinical trials and regulatory approval (Fleming, 2018). Using the AI technologies, the drug discovery process will speed up the drugs that are released into the market with short period of time, desired dose, overall safety, efficacy and other parameters required according to the individual patient need (Bender \& Cortes-Ciriano, 2020; Paul et al., 2021).

The authors have identified the potential role of artificial intelligence in both disease and drug discovery process. The AI tools used for drug discovery process are highlighted in this manuscript. This article is intended for all researchers and academicians who studies about the applications of artificial intelligence related to medicine and health care. Considering the facts, the paper is arranged as follows. The review of literature is provided in Literature Survey in which the SARS$\mathrm{CoV}-2$ detection methods, artificial intelligence role in disease identification and drug discovery are discussed. In another part the features of artificial intelligence, role of artificial intelligence for disease diagnosis and drug discovery and finally conclusions are addressed.

\section{SURVEY METHODOLOGY}


90 The electronic searches were performed to retrieve literature in journal databases such as Google 91 Scholar, Nature, WHO and Pub Med. A list of search terms can be seen in Table S1. The search

92 terms used for collecting the data are SARS-CoV-2 disease, pathology, identification methods, RT-PCR, Immunoassay, artificial intelligence, drug discovery using artificial intelligence, machine learning, computed tomography, radiology, ophthalmology and COVID-19 disease data. The qualitative and quantitative articles were retrieved from literature and the insights related to COVID-19 disease diagnosis, measurable data to formulate the facts related to drug discovery aspects in research was highlighted. The following types of studies are included such as review articles, research papers and short communications. The research papers with only abstract, books and conference papers are excluded. The information provides the current knowledge by identifying the gaps in the literature, different theoretical perspectives and also suggests the future directions for research. All authors assessed the cited studies for quality of the information.

\section{LITERATURE SURVEY}

Mei et al. (2020) discussed how AI enabled diagnosis of COVID-19. In this study, AI algorithms were used to detect the disease based on chest findings, laboratory tests, exposure history and clinical symptoms. A total of 905 patients were tested using RT-PCR, and of them, 279 patients were diagnosed using AI. In 905 tested cases, 419 patients (46.3\%) were positive for COVID-19 infection (Mei et al., 2020). AI system correctly identified 17 of 25 patients who had CT scans and tested positive for COVID-19 using RT-PCR. The researchers in this study highlighted the importance of AI to accelerate COVID-19 diagnosis (Mei et al., 2020).

Dhamad \&Rida, 2020 has given insights about the serological and molecular methods for detection of COVID-19. In case of molecular methods RT-PCR, CRISPR-Cas 12 based method and isothermal amplification-based approaches are used for detection of COVID-19 where as in serological methods lateral flow assay and ELISA are used for detection of COVID-19. Molecular method RT-PCR is the gold standard method for detection of COVID-19 with accuracy.

Porte et al., 2021 evaluated the performance of two fluorescence immunoassays SOFIA SARS Antigen FIA and STANDARD F COVID-19 Antigen FIA. The two FIA kits showed 100\% 
118 sensitivity and $96.9 \%$ specificity which indicates that the FIA kits can be used as diagnostic tools

119 for early stages of COVID-19 infection other than RT-PCR.

121 In recent study by Vaishya et al. (2020) also highlighted the importance of AI and its applications 122 for COVID-19. Eight different and significant applications for COVID-19 were discussed 123 including early detection of disease, monitoring the treatment of patients, tracing the primary and 124 secondary contacts of COVID-19-positive cases, projection of cases and mortality, drug 125 development, vaccine development, reduction of workload on health care workers and finally 126 disease prevention (Vaishya et al., 2020). This study provides support for the use of AI in detecting 127 and managing disease in near future (Vaishya et al., 2020).

128 Li et al. (2020) evaluated the diagnostic accuracy of detecting COVID-19 disease and community129 acquired pneumonia using pulmonary CT. In this study, deep learning technology was used to 130 identify community-acquired COVID-19 on chest CT scans. This study used a multidisciplinary 131 approach to diagnose the disease (Li et al., 2020).

132 Lalmuanawma et al. (2020) discussed about AI and machine learning tools applications in 133 COVID-19. The AI tools used for screening, prediction, contact tracing and drug development for 134 COVID-19. Mohanty et al. (2020) highlighted the importance of drug repurposing for COVID-19 using AI. Deep learning algorithms can help in predicting potential antiviral drug molecules for COVID-19.

137

In a study reported by Stokes et al., 2020, machine learning techniques were used in drug discovery process. Deep neural networks are used for predicting the inhibitor molecule for disease causing agents. Although machine learning tools are available there is a need to discover and study the proteins of interest in disease and drug discovery process.

Allam et al. (2020) reported on a health policy for detection of COVID-19 using an AI tool that can be applied internationally. In China, the companies Blue dot and Meta biota developed AI algorithms for accurate diagnosis of COVID-19. Ho (2020) discussed drug combinations for COVID-19, whose results must be evaluated to develop antiviral therapy for COVID-19. Drug 
145 146

147 148

combinations are screened and evaluated for drug dose compositions for personalized therapy are optimized using AI.

Das et al. (2020) highlighted the importance of open source machine learning tool for prediction of mortality risk among COVID-19 patients. The machine learning algorithm such as logistic regression, k-nearest neighbor, support vector machine, gradient boosting and random forest were used to analyze the mortality rate and found that logistic regression algorithm was the best one for mortality risk prediction.

In recent study by Kumar et al., 2021 highlighted the importance of artificial intelligence in diagnosis and prediction of COVID-19 disease. Further, the data sets and visualization techniques for COVID-19, designing of drugs for COVID-19 using artificial intelligence and role of deep learning and machine learning tools were discussed.

\section{FEATURES OF AI}

AI programming tools can help to identify signs of certain diseases (Caruso et al., 2020). Large volumes of health data are collected to identify a particular disease, including magnetic resonance images, X-ray results, CT scans, vaccinations, skin lesions, eye images, DNA sequences, blood samples, past medical history and current medications (Hosny et al., 2018) (Figure 1). However, AI programming tools must be validated in large groups of people. Machine learning tools particularly deep learning algorithms have been used to diagnose disease automatically, which makes diagnosis cheaper and more accessible (Davenport \& Kalakota, 2019). Using the data, the algorithms can draw conclusions in fraction of seconds and these can be accessed more easily for disease diagnosis. The process is inexpensive and less time-consuming for identifying disease compared with traditional methods of diagnosis (Agrebi \& Larbi, 2020). In the case of COVID19, two companies called Blue Dot and Meta biota (Allam et al., 2020) demonstrated the role of AI in early detection of SARS-CoV-2; Blue Dot predicted the outbreak areas in China, and Meta biota used big data analytics to count the outbreaks globally (Allam et al., 2020). In India, qure.ai algorithm tool has been used successfully to interpret the radiology images for diagnosis COVID-

19and tuberculosis. The X-ray screening tool qXR (deep learning tool) was used for diagnostic and critical care management. Q Scout is another algorithm tool helps in contact monitoring of 
174 COVID-19patients (https://qure.ai). RadVid-19 is the algorithm tool used by the Brazilian doctors

175 to analyze chest X-rays and CT scans to find the spots in lungs which are markers of infection due

176 to SARS-CoV-2 (https://radvid-19.com). Deep COVID-XR is the deep learning algorithm tool

177 used for detecting COVID-19 in patients based on chest radiographs by US clinical radiologists

178 during pandemic period (Wehbe et al., 2020). Elzeki et al., 2021 evaluated the performance of 179 CXRVN (Chest X-ray COVID Network) deep learning computer aided model for early detection 180 of COVID-19. In this model three different datasets were used and compared with pretrained 181 models viz. Google net, Alex Net and Res Net. The evaluation results showed 94.5\% accuracy for 182 the proposed CXRVN model.

Figure 1. Illustration of how artificial intelligence uses the health data to identifying the disease. (Source:https://www.europeanpharmaceuticalreview.com/news/68136/flu-vaccine-laeukemia; https: //www.geeksforgeeks.org/top-5-trends-in-artificial-intelligence-that-may-dominateeconomictimes.indiatimes.com/magazines/panache/blood-test-can-predictseverity-of-covid-19; https://www.canstockphoto.com/ Patient medical history form17652858.html).

\section{COVID -19 DETECTION METHODS}

\section{RT-PCR}

195

196

197

Currently the corona virus SARS CoV-2 is detected using methods like RT-PCR, Rapid test kits, and CT scanning. In these methods, RT-PCR is preferred one and also called as gold standard method (Carter et al., 2020). In this method nasopharyngeal swab or oral pharyngeal swab is collected and kept in viral transport medium (Lopez et al., 2019). The genetic material i.e. RNA is extracted and converted into cDNA using reverse transcriptase enzyme. The cDNA further undergoes PCR amplification involves denaturation, annealing, extension and cleavage (Udugama et al., 2020). The DNA probe is used and in amplification process and it consists of reporter dye 
202 and quencher dye at 5' end and 3' end respectively. The reporter dye gets multiplied during 203 amplification process, excited and leads to emission upon focusing a beam of light (fluorescence).

204 The final step of this RT-PCR technique is conversion of light into digital data (Carter et al., 2020).

205 The formation of graphs with cycle on x-axis and fluorescence intensity on y-axis is formed. The 206 data is analyzed and considered as COVID positive or negative (Figure 2). However, this method 207 takes 2 days to release the output. In present adverse conditions there is undersupply of the RT208 PCR kits. In order to renew this kit, something advanced and accurate tool are required whose 209 response is efficient (Udugama et al., 2020).

210 Figure 2. Illustration of RT-PCR Assay protocol for detection of COVID-19. The swab 211 collected from suspected person is used for RNA extraction, conversion to cDNA, PCR 212 amplification and finally fluorescence light data conversion into digital data. The digital 213 graph describes the test sample is COVID-19 positive/negative. (Source: 214 http://www.fredhutch.org /en/research/diseases/coronavirus/serology-testing.html; 215 http://www.researchamerica.org/bl og/explained-how-identify-active-covid-19-infection216 people; http://www.medmastery.com/ guide/covid-19-clinical-guide/how-rt-pcr-used-test217 covid-19; www.advanced-biotechs.com/ quantstudio

\section{IMMUNOASSAY METHOD}

219 The other method is immunoassay which is rapid test kit. In this, the immunoglobulins IgG and 220 IgM antibodies are measured against the SARS-COV-2 proteins. The method is considered as fast 221 and precise as it produces output in 10-15 minutes by examining whole blood (Jacofsky et al., 222 2020). In this the blood sample is collected and tested in which red colored lines are formed 223 opposite to their respective sites as shown in Figure 3. The presence of IgG and IgM antibodies 224 results in the formation of red colored lines against ' $G$ ' site and ' $M$ ' site respectively (Jacofsky et 225 al., 2020). Formation of red lines at the minimum of two confirms that the sample is having SARS$226 \mathrm{COV}-2$ proteins. However, this technique is not specific as it cannot detect virus in the incubation 227 period and may show false negative result which leads to adverse effect in future (Udugama et al., 228 2020).

Figure 3. Immunoassay detection of COVID-19 disease. 
231 CT (Computed Tomography) is a sensitive medical imaging technique used for identification of

232 diseases. It involves imaging the organs with the help of computational knowledge (Shi et al., 233 2020). AI algorithms are used to enhance the chest CT findings for rapid diagnosis. The method 234 is sensitive as it captures every small detail of the lungs. For detection of COVID-19, the imaging 235 features used are ground glass opacities, consolidations and crazy paving patterns (Ozturk et al., 236 2020). Considering the public health perspective and the need to quickly isolate patients with 237 COVID-19, chest CT is used because it is more sensitive than chest radiography and other 238 techniques. However, these methods can be used in combination for accurate and better results 239 and can be accelerated with new AI algorithms (Xu et al., 2020). AI accelerates the process 240 compared to conventional techniques and does not involve symptomatic treatment. Daily updates

241 from patients can be studied, and the rate of infection can be inspected by observing each 242 movement of an infected person (Davenport \& Kalakota, 2019). A recent study by Li \& Xia (2020) 243 reported that $\mathrm{CT}$ scan data was analyzed for 51 patients and found good results. Hence, CT scan 244 data can be used for rapid diagnosis of COVID-19. The AI-based mobile app AI4COVID-19 was developed for evaluating COVID-19 patients based on cough samples (Imran et al., 2020).

246

247

248

249

250

251

252

253

254

255

256

257

258

259

260

\section{ARTIFICIAL INTELLIGENCE IN DRUG DISCOVERY}

The use of AI in pharmacogenomics and drug development quickens the process of drug production. Drug development and market release takes at least 14 years, but AI uses an in-silico approach, which makes in vitro and in vivo tests easier (Zhavoronkov et al., 2020). The first step in drug discovery using AI is to set a hypothesis. Next the hypothesis is validated by selecting potential lead molecules from the lead database. The activity of the lead molecules is evaluated using in-vitro and in-vivo tests (Vamathevan et al., 2019). The potential lead molecule is said to be a drug after optimizing parameters like affinity to bind the target, adverse effects, efficacy, bioavailability and therapeutic effect (Batool et al., 2019; Chan et al., 2019). The potential lead molecule from thousands of compounds is identified using neural networks, homology modeling, protein fold prediction, omics analytics, SMILES, LSTM models, etc. (Figure 4) (Yang et al., 2019). The drug toxicity is essential to prevent the side effects and toxic effects in humans. The web-based tools used for evaluating the toxicity are Deep Tox, Lim Tox, Tox 21 and admetSAR (Cheng et al., 2012; Yang et al., 2018). In COVID-19 disease, the enzyme 3CLpro can be considered as a target that is inhibited by a lead molecule. De novo design tools would be used to 
261 obtain a potential drug candidate for COVID-19 (Amin et al., 2020). The AI tools used in drug 262 discovery are PADME, MANTRA, PREDICT, deep neural Net QSAR, Deep chem, Deep Tox, 263 XenoSite, SMARTCyp, FAME, ORGANIC, Potential Net, Hit Dexter, Delta Viva, Neural graph 264 finger print, Alpha fold and Chemputer (Paul et al., 2021).

265 However, advanced and new algorithms are required to substitute the human brains in near future.

266 Collaboration among biotechnology, pharmaceutical and AI companies could streamline research 267 and development efforts to identify novel, rare drug molecules and also personalized medicine.

Figure 4. Steps involved in artificial intelligence for drug development

269

270

271

272

273

274

275

276

277

278

279

280

281

282

283

284

285

286

287

\section{CONCLUSION}

The AI supports the future needs of medicine and health care by accelerating disease identification and drug discovery process. The technology develops the machine learning tools in health care particularly in the area of emerging diseases like COVID-19, regenerative medicine, gene therapy and pharmacology. Further, the artificial intelligence entails personalized medicine, assisted diagnostics, biomarkers, drug discovery and development. However, the advanced and new algorithms are required to substitute the human brains in near future. This enables an opportunity to collaborate biotech, pharma and artificial intelligence companies to streamline their research and development to identify the novel, rare drug molecules and also personalized medicine.

\section{REFERENCES}

1. Abiodun OI, Jantan A, Omolara AE, Dada KV, Mohamed NA, \& Arshad H. 2018. Stateof-the-art in artificial neural network applications: A survey. Heliyon 4(11), e00938 DOI 10.1016/j.heliyon. 2018. e00938.

2. Abiodun OI, Jantan A, Omolara AE, Dada KV, Umar AM, Linus OU, \& Kiru MU. 2019. Comprehensive review of artificial neural network applications to pattern recognition. IEEE Access 7:158820-158846. DOI 10.1109/ACCESS.2019.2945545.

3. Agrawal P. 2018. Artificial intelligence in drug discovery and development. J Pharmacovigilance 6(2) 1000e173 DOI 10.4172/2329-6887.1000e173. 
4. Agrebi S \& Larbi A. 2020. Use of artificial intelligence in infectious diseases. In Artificial Intelligence in Precision Health 415-438 DOI 10.1016/B978-0-12-817133-2.00018-5.

5. Ahuja AS. 2019. The impact of artificial intelligence in medicine on the future role of the physician. Peer J 7: e7702 DOI 10.7717/peerj.7702.

6. Allam Z, Dey G \& Jones DS. 2020. Artificial intelligence (AI) provided early detection of the coronavirus (COVID-19) in China and will influence future Urban health policy internationally. AI 1(2): 156-165 DOI 10.3390/ai1020009.

7. Amin SA, Ghosh K, Gayen S \& Jha T. 2020. Chemical-informatics approach to COVID19 drug discovery: Monte Carlo based QSAR, virtual screening and molecular docking study of some in-house molecules as papain-like protease (PLpro) inhibitors. Journal of Biomolecular Structure and Dynamics 1-10 DOI 10.1080/07391102.2020.1780946.

8. Batool M, Ahmad B \& Choi S. 2019. A structure-based drug discovery paradigm. International journal of molecular sciences 20(11): 2783 DOI 10.3390/ijms20112783.

9. Bender A, \& Cortes-Ciriano I. 2020. Artificial intelligence in drug discovery: what is realistic, what are illusions? Part 1: Ways to make an impact, and why we are not there yet. Drug Discovery Today 26(2): 511-524 DOI 10.1016/j.drudis.2020.12.009.

10. Bohr A, \& Memarzadeh K. 2020. The rise of artificial intelligence in healthcare applications. In Artificial Intelligence in Healthcare 25-60 Academic Press DOI 10.1016/B978-0-12-818438-7.00002-2.

11. Carter LJ, Garner LV, Smoot JW, Li Y, Zhou Q, Saveson CJ, \& Jervey SR. 2020. Assay techniques and test development for COVID-19 diagnosis. ACS Cent. Sci. 6:591-605 DOI 10.1021/acscentsci.0c00501.

12. Caruso D, Zerunian M, Polici M, Pucciarelli F, Polidori T, Rucci C, \& Laghi A. 2020. Chest CT features of COVID-19 in Rome, Italy. Radiology 296: E79-E85 DOI 10.1148/radiol.2020201237.

13. Chan HS, Shan H, Dahoun T, Vogel H \& Yuan S. 2019. Advancing drug discovery via artificial intelligence. Trends in pharmacological sciences 40(8): 592-604 DOI 10.1016/j.tips.2019.06.004.

14. Cheng F, Li W, Zhou Y, Shen J, Wu Z, Liu G, \& Tang Y. 2012. admet SAR: a comprehensive source and free tool for assessment of chemical ADMET properties. J. Chem. Inf. Model 52(11): 3099-3105 DOI 10.1021/ci300367a. 
319

320

321

322

323

324

325

326

327

328

329

330

331

332

333

334

335

336

337

338

339

340

341

342

343

344

345

346

347

348

15. Das AK, Mishra S, \& Gopalan SS. 2020. Predicting CoVID-19 community mortality risk using machine learning and development of an online prognostic tool. Peer J 8: e10083 DOI 10.7717/peerj.1008.

16. Dhamad AE, \& Rhida MAA. 2020. COVID-19: molecular and serological detection methods. Peer J 8: e10180 DOI 10.7717/peerj.10180.

17. Davenport T \& Kalakota R. 2019. The potential for artificial intelligence in healthcare. Future healthcare journal 6(2): 94-98 DOI 10.7861/futurehosp.6-2-94.

18. Elzeki OM, Shams M, Sarhan S, Abd Elfattah M, \& Hassanien AE. 2021. COVID-19: a new deep learning computer-aided model for classification. Peer J Computer Science 7: e358. DOI 10.771/peerj-cs.358.

19. Fleming N. 2018. How artificial intelligence is changing drug discovery. Nature 557 (7706): S55-S57 DOI 10.1038/d41586-018-05267-x.

20. Ho D. (2020). Addressing COVID-19 Drug Development with Artificial Intelligence. Advanced Intelligent Systems 2 (2000070): 1-4 DOI 10.1002/aisy.202000070.

21. Hosny A, Parmar C, Quackenbush J, Schwartz LH, \& Aerts HJ. 2018. Artificial intelligence in radiology. Nature Reviews Cancer 18(8): 500-510 DOI 10.1038/s41568018-0016-5.

22. https://economictimes.indiatimes.com/magazines/panache/blood-test-can-predictseverity-of-covid-19

23. https://qure.ai

24. https://radvid-19.com

25. https://www.canstockphoto.com/Patient medical history form-17652858.html

26. https://www.europeanpharmaceuticalreview.com/news/68136/flu-vaccine-laeukemia

27. http://www.fredhutch.org/en/research/diseases/coronavirus/serology-testing.html;

28. https://www.geeksforgeeks.org/top-5-trends-in-artificial-intelligence-that-may-dominate$\underline{2020 \mathrm{~s} /}$

29. http://www.medmastery.com/guide/covid-19-clinical-guide/how-rt-pcr-used-test-covid$\underline{19}$

30. http://www.researchamerica.org/blog/explained-how-identify-active-covid-19infection-people 
31. Imran A, Posokhova I, Qureshi HN, Masood U, Riaz S, Ali K, \& Nabeel M. 2020. AI4COVID-19: AI enabled preliminary diagnosis for COVID-19 from cough samples via an app. Informatics in Medicine Unlocked 20:100378 DOI 10.1016/j.imu.2020.100378.

32. Jacofsky D, Jacofsky EM, \& Jacofsky M. 2020. Understanding antibody testing for covid19. The Journal of Arthroplasty 35(7): S74-S81 DOI 10.1016/j.arth.2020.04.055.

33. Jiang F, Jiang Y, Zhi H, Dong Y, Li H, Ma S, \& Wang Y. 2017. Artificial intelligence in healthcare: past, present and future. Stroke and vascular neurology 2: e000101 231-243 DOI 10.1136/svn-2017-000101.

34. Kumar V, Singh D, Kaur M, \& Damasevicius R. 2021. Overview of current state of research on the application of artificial intelligence techniques for COVID-19. Peer J computer science 7: e564 DOI 10.7717/peerj-cs-564.

35. Lalmuanawma S, Hussain J, \& Chhakchhuak L. 2020. Applications of machine learning and artificial intelligence for Covid-19 (SARS-CoV-2) pandemic: A review. Chaos, Solitons, and Fractals, 139: 110059. DOI 10.1016/j.chaos.2020.110059.

36. Li L, Qin L, Xu Z, Yin Y, Wang X, Kong B, \& Cao K. 2020. Artificial intelligence distinguishes COVID-19 from community acquired pneumonia on chest CT. Radiology 200905 DOI 10.1148/radiol.2020200905.

37. Li Y \& Xia L. 2020. Coronavirus disease 2019 (COVID-19): role of chest CT in diagnosis and management. American Journal of Roentgenology 214(6):1280-1286 DOI 10.2214/AJR.20.22954.

38. Lopez SM, Martin JM, Johnson M, Kurs-Lasky M, Horne WT, Marshall CW, \& Shaikh N. 2019. A method of processing nasopharyngeal swabs to enable multiple testing. Pediatric research 86(5): 651-654 DOI 10.1038/s41390-019-0498-1.

39. Mohanty S, Rashid MHA, Mridul M, Mohanty C, \& Swayamsiddha S. 2020. Application of Artificial intelligence in COVID-19 drug repurposing. Diabetes \& Metabolic Syndrome: Clinical Research \& Reviews 14(5): 1027-1031 DOI 10.1016/j.dsx.2020.06.068.

40. Mohs RC, \& Greig NH. 2017. Drug discovery and development: Role of basic biological research. Alzheimer's \& Dementia: Translational Research 3(4): 651-657 DOI 10.1016/J.TRCI.2017.10.005. 
378

379

380

381

382

383

384

385

386

387

388

389

390

391

392

393

394

395

396

397

398

399

400

401

402

403

404

405

406

407

41. Mei X, Lee HC, Diao KY, Huang M, Lin B, Liu C, \& Bernheim A. 2020. Artificial intelligence-enabled rapid diagnosis of patients with COVID-19. Nature Medicine 26: 1224-1228 DOI 10.1038/s41591-020-0931-3.

42. Nguyen G, Dlugolinsky S, Bobak M, Tran V, García ÁL, Heredia I, \& Hluchy L. 2019. Machine learning and deep learning frameworks and libraries for large-scale data mining: a survey. Artificial Intelligence Review 52(1): 77-124 DOI 10.1007/s10462-018-09679-z.

43. Paul D, Sanap G, Shenoy S, Kalyane D, Kalia K, \& Tekade RK. 2021. Artificial intelligence in drug discovery and development. Drug Discovery Today. 26(1): 80-93 DOI 10.1016/j.drudis.2020.10.010.

44. Porte L, Legarraga P, Iruretagoyena M, Vollrath V, Pizarro G, Munita J, \& Weitzel T. 2021. Evaluation of two fluorescence immunoassays for the rapid detection of SARSCoV-2 antigen - new tool to detect infective COVID-19 patients. Peer J 9: e10801 DOI 10.7717/peerj.10801.

45. Riaz A, Rasul A, Sarfraz I, Nawaz J, Sadiqa A, Zara R, \& Selamoglu Z. 2020. Chemical Biology Toolsets for Drug Discovery and Target Identification. In Cheminformatics and its Applications. Intech Open DOI 10.5772/intechopen.91732.

46. Ozturk T, Talo M, Yildirim EA, Baloglu UB, Yildirim O \& Acharya UR. 2020. Automated detection of COVID-19 cases using deep neural networks with X-ray images. Computers in Biology and Medicine 121: 103792 DOI 10.1016/j.compbiomed.2020.103792.

47. Shi F, Wang J, Shi J, Wu Z, Wang Q, Tang Z, \& Shen D. 2020. Review of artificial intelligence techniques in imaging data acquisition, segmentation and diagnosis for covid19. IEEE Reviews in Biomedical Engineering 14: 4-15 DOI 10.1109/RBME.2020.2987975.

48. Singhal T. 2020. A review of coronavirus disease-2019 (COVID-19). The Indian Journal of Pediatrics 87(4): 281-286 DOI 10.1007/s12098-020-03263-6.

49. Stokes JM, Yang K, Swanson K, Jin W, Cubillos-Ruiz A, Donghia NM, MacNair CR, French S, Carfrae LA, Bloom-Ackerman Z. 2020. A Deep Learning Approach to Antibiotic Discovery. Cell 180: 688-702 DOI 10.1016/j.cell.2020.01.021.

50. Tahamtan A, \& Ardebili A. 2020. Real-time RT-PCR in COVID-19 detection: issues affecting the results 20(5):453-454 DOI 10.1080/14737159.2020.1757437. 
408

409

410

411

412

413

414

415

416

417

418

419

420

421

422

423

424

425

426

427

428

429

430

431

432

433

434

435

436

437

51. Udugama B, Kadhiresan P, Kozlowski HN, Malekjahani A, Osborne M, Li VY, \& Chan WC. 2020. Diagnosing COVID-19: the disease and tools for detection. ACS nano, 14(4): 3822-3835 DOI 10.1021/acsnano.0c02624.

52. Vamathevan J, Clark D, Czodrowski P, Dunham I, Ferran E, Lee G, \& Zhao S. 2019. Applications of machine learning in drug discovery and development. Nature Reviews Drug Discovery 18(6): 463-477 DOI 10.1038/s41573-019-0024-5.

53. Vaishya R, Javaid M, Khan IH, \& Haleem, A (2020). Artificial Intelligence (AI) applications for COVID-19 pandemic. Diabetes \& metabolic syndrome 14(4): 337-339 DOI 10.1016/j.dsx.2020.04.012.

54. Wehbe RM, Sheng J, Dutta S, Chai S, Dravid A, Barutcu S, \& Katsaggelos AK. 2020. Deep COVID-XR: An Artificial Intelligence Algorithm to Detect COVID-19 on Chest Radiographs Trained and Tested on a Large US Clinical Dataset. Radiology 299: E167E176 DOI 10.1148/radiol.2020203511.

55. Wu R, Wang L, Kuo HCD, Shannar A, Peter R, Chou PJ, Poiani GJ. 2020. An update on current therapeutic drugs treating COVID-19. Current Pharmacology Reports 1-15 DOI $10.1007 / \mathrm{s} 40495-020-00216-7$

56. www.advanced-biotechs.com/quantstudio.

57. Xu B, Xing Y, Peng J, Zheng Z, Tang W, Sun Y, \& Peng F. 2020. Chest CT for detecting COVID-19: a systematic review and meta-analysis of diagnostic accuracy. European Radiology 30(10): 5720-5727 DOI 10.1007/s00330-020-06934-2.

58. Yang H, Sun L, Li W, Liu G, \& Tang Y. 2018. In silico prediction of chemical toxicity for drug design using machine learning methods and structural alerts. Frontiers in chemistry 6 : 30 DOI 10.3389/fchem.2018.00030.

59. Yang X, Wang Y, Byrne R, Schneider G \& Yang S. 2019. Concepts of artificial intelligence for computer-assisted drug discovery. Chemical reviews 119(18): 10520-10594 DOI 10.1021/acs.chemrev.8b00728.

60. Zhavoronkov A, Vanhaelen Q \& Oprea TI. 2020. Will Artificial Intelligence for Drug Discovery Impact Clinical Pharmacology? Clinical Pharmacology \& Therapeutics 107(4): 780-785 DOI 10.1002/cpt.1795.

PeerJ reviewing PDF | (2020:07:51371:3:0:NEW 19 Jul 2021) 


\section{Figure 1}

Illustration of how artificial intelligence uses the health data to identifying the disease.

The data related to CT scan, MRI, X-ray, skin lesions, eye, blood tests, patient medical

history, medicines, vaccines and genetic information applied for diagnosing the disease using artificial intelligence (Source:

https://www.europeanpharmaceuticalreview.com/news/68136/flu-vaccine-laeukemia ; https:

//www.geeksforgeeks.org/top-5-trends-in-artificial-intelligence-that-may-dominate-2020s/ ;https:// economictimes.indiatimes.com/magazines/panache/blood-test-can-predict-severityof-covid-19; https://www.canstockphoto.com/ Patient medical history form-17652858.html). 


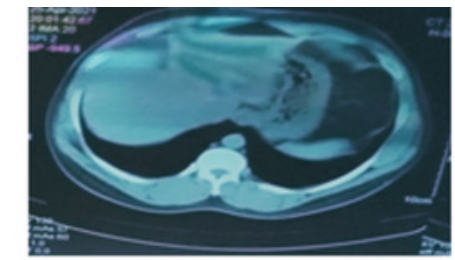

CT Scan image

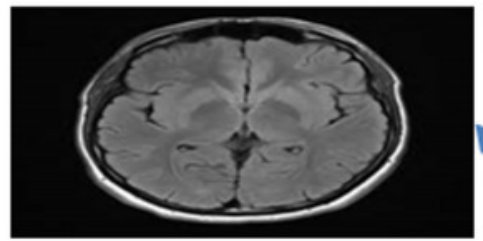

MRI image

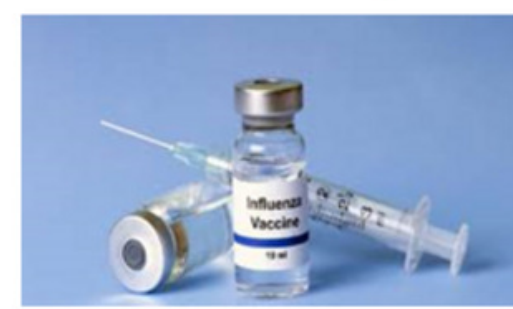

Vaccination schedule

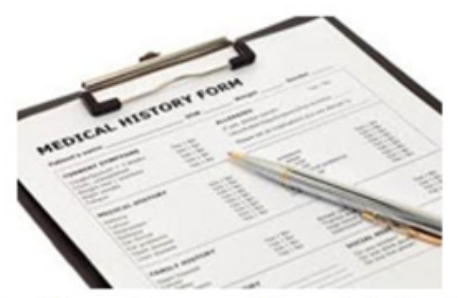

Patient medical history
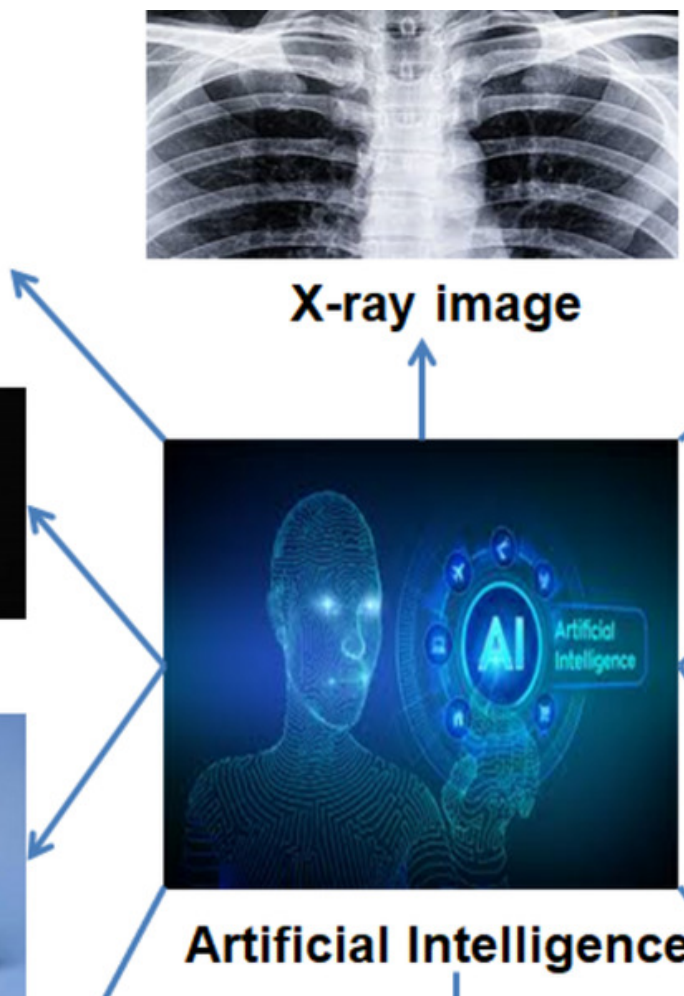

Artificial Intelligence

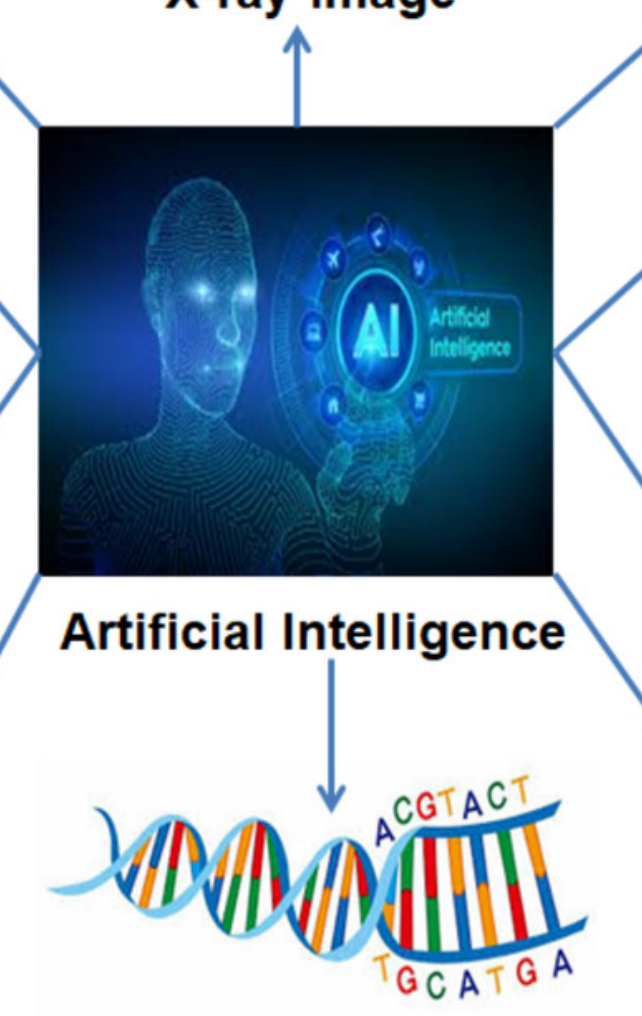

Genetic information

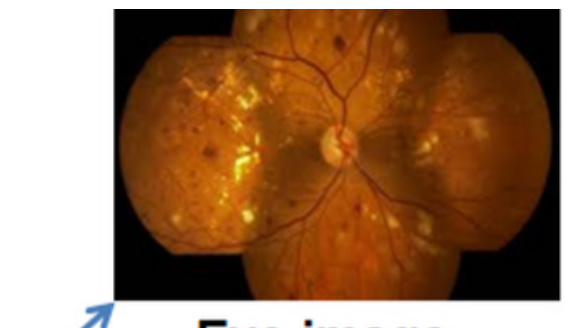

Eye image

\section{Medicines}




\section{Figure 2}

Illustration of RT-PCR Assay protocol for detection of COVID-19.

The swab collected from suspected person is used for RNA extraction, conversion to CDNA, PCR amplification and finally fluorescence light data conversion into digital data. The digital graph describes the test sample is COVID-19 positive/negative
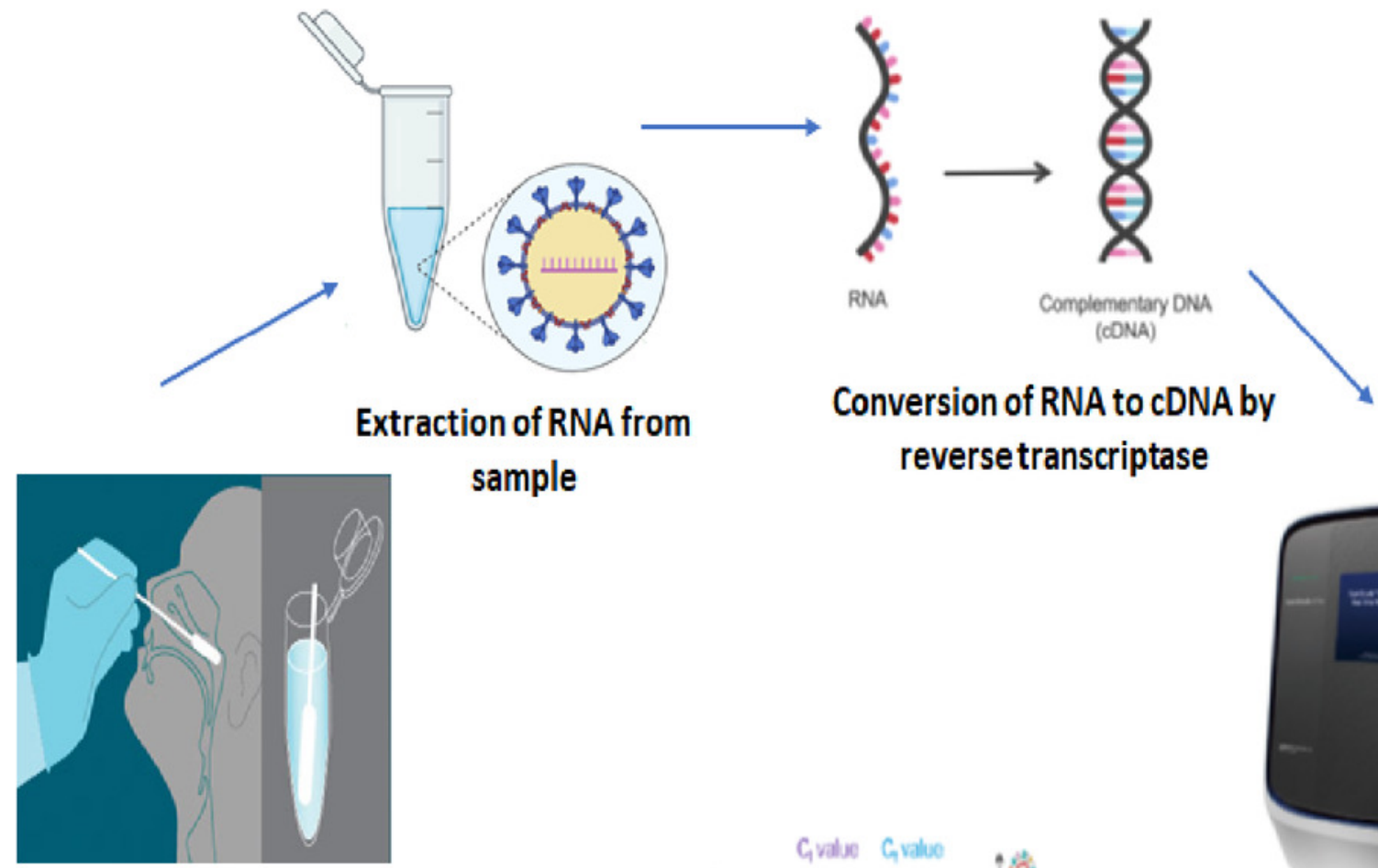

Collection of swab from suspected person

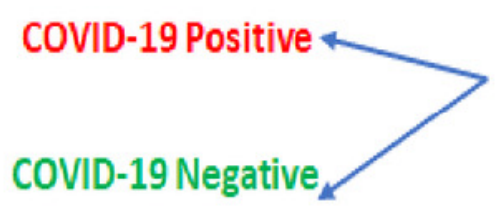

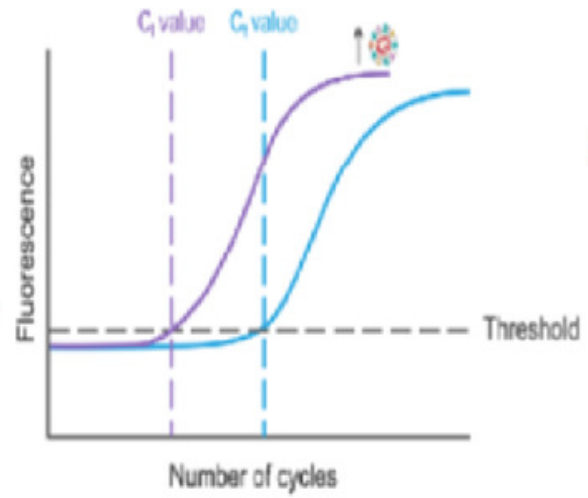

Conversion of Fluorescence data into digital data 
Figure 3

Immunoassay detection of COVID-19 disease.

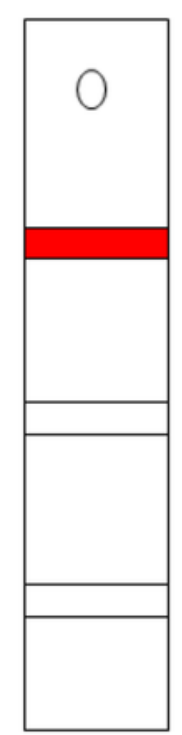

Negative

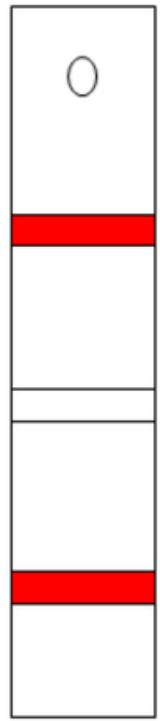

\section{Positive}

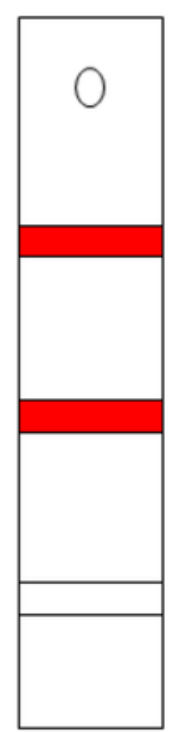

\section{Positive}

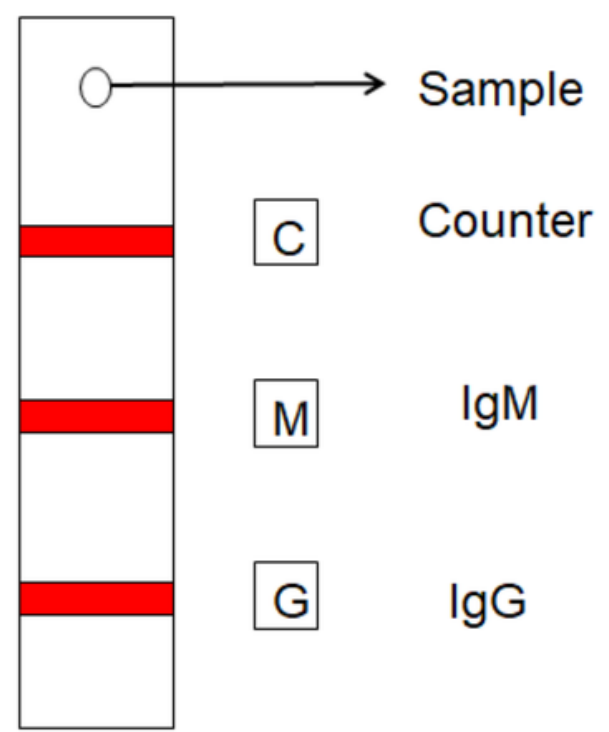

Positive 
Figure 4

Steps involved in artificial intelligence for drug development.

Artificial Intelligence

1. Neural networks

2. Machine learning tools

3. Repurposing drug candidates

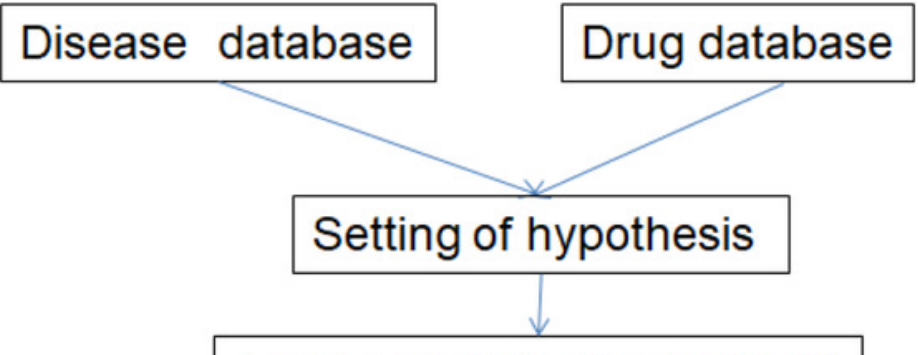

from lead compounds database

In-vitro and in-vivo tests

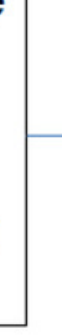
validation

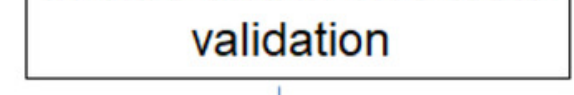

Potential lead molecule

Drug trails

Drug approval
Pharmacokinetic and pharmacological features evaluated:

- Affinity to bind to the target

- Efficacy of the drug

- Therapeutic affect of the drug

- Bioavailability of the drug

- Adverse effects of the drug 